\title{
Alpelisib Inhibits PIK3CA-Mutant Breast Cancer Growth Through AKT-Dependent Bim Induction and Mcl-1 Degradation
}

\section{Xiao Tan}

Xiangya Hospital Central South University

\section{Zhongqiang Zhang}

Second Xiangya Hospital

Ping Liu

Xiangya Hospital Central South University

Hongliang Yao

Second Xiangya Hospital

jingshan tong ( $\nabla$ tongjingshan@gmail.com )

UPMC Hillman Cancer Center

\section{Research}

Keywords: Alpelisib, PIK3CA, Breast cancer, Bim, Mcl-1

Posted Date: July 2nd, 2020

DOl: https://doi.org/10.21203/rs.3.rs-38718/v1

License: (c) (1) This work is licensed under a Creative Commons Attribution 4.0 International License. Read Full License 


\section{Abstract}

Background: PIK3CA mutations are common genomic alterations in estrogen receptor (ER)-positive breast cancers, currently, the development of selective PI3Ka (phosphatidylinositol 3-kinase a) inhibitors is ongoing. The mechanisms contributing to the anticancer activity of alpelisib in PIK3CA-mutant breast cancer cells and the mechanism of acquired resistance to alpelisib remain elusive.

Methods: Drug-sensitive cell lines were exposed to alpelisib to establish alpelisib-resistant cell lines. Western blotting was used to assess changes in protein expression. Apoptosis was evaluated by flow cytometry. In vivo with mouse xenograft models and in vitro colony formation and MTS and assay were carried out to determine the growth inhibitory effects of the tested drugs. Protein half-lives were examined and proteasome inhibitors were used to estimate protein degradation. Gene knockdown was carried out using shRNA or siRNA.

Results: In the present study, we report the potent induction of apoptosis by alpelisib in PIK3CA-mutant breast cancer cell lines. AKT phosphorylation suppression, AKT/Foxo3a-dependent Bim induction, and AKT/GSK-3ß-dependent Mcl-1 degradation were observed. Apoptosis induced by alpelisib was attenuated by Mcl-1 (4A) overexpression or Bim suppression. Furthermore, alpelisib could not modulate Mcl-1 or Bim levels in cell lines that were resistant to alpelisib. AKT inhibitor and alpelisib combination restored the sensitivity of alpelisib-resistant cells to growth inhibition and apoptosis in vitro and in vivo.

Conclusions: Therefore, modulation of Mcl-1 degradation and AKT-dependent Bim induction are crucial for mediating the resistance and sensitivity of PIK3CA-mutant breast tumor cells to alpelisib, thus making it a productive strategy for overcoming acquired resistance to alpelisib.

\section{Background}

In both healthy and malignant cells, the PI3K (phosphatidylinositol 3-kinase) pathway is a key driver of survival, metabolism, and growth $(1,2)$. More than two-thirds of breast cancers have PI3K pathway activation via HER2 amplification, PTEN tumor suppressor deletion, or PIK3CA mutations causing oncogenesis $(3,4)$. Class IA PI3Ks are a group of holoenzymes that have $\mathrm{p} 85$, a regulatory subunit attached to any of three potential catalytic subunits, p110a (encoded by PIK3CA), p1108, and p110 $\beta$ subunits $(5,6)$. To be activated, each class IA PI3K is recruited to phosphotyrosine peptides, such as those on RTKs (receptor tyrosine kinases) and adaptors (e.g., IRS-1), via p85 SH2 domains (7, 8). p110 3 can also be activated by GPCRs (G protein-coupled receptors)(9). The function of class I PI3Ks involves phosphorylating the inositol ring of PI(4,5)P2 (phosphatidylinositol (4,5)-bisphosphate) at the D-3 position to generate the $2^{\text {nd }}$ messenger PIP3 (phosphatidylinositol $(3,4,5)$-trisphosphate)(10). PIP3 recruits its effectors (e.g., AKT) to the membrane through their $\mathrm{PH}$ (pleckstrin homology) domains and activates them $(11,12)$. Thus, inhibiting PI3K activity may be a potential approach to treating breast cancer, and several such agents are entering clinical trials. 
Currently, selective inhibitors of PI3Ka (PI3K p110a) are being clinically evaluated in patients with malignancies in their stage $(13,14)$. Promising results were observed in breast tumor patients harboring PIK3CA mutations $(15,16)$. There were some benefits of these agents, but typical relapse was observed even in patients who initially had a positive response after a month of treatment(17). With the ER-positive nature of the majority of PIK3CA-mutant tumors, it may be stipulated that these cells can survive through both pathways governing proliferation and survival(18). The proof of the cooperation of the ER and PI3K pathways in the advancement of tumors arose from a clinical study $(19,20)$. A remarkable improvement was observed in the progressive defense of ER-positive breast tumor patients who underwent with everolimus treatment, an mTOR inhibitor, combined with exemestane, an inhibitor for the anti-estrogen aromatase $(21,22)$.

The small, orally bioavailable molecule, alpelisib (BYL719), is an a-specific PI3K inhibitor that specifically inhibits $\mathrm{p} 110 \mathrm{a}$ at a strength nearly 50 -fold that of other isoforms(22). Alpelisib was FDA approved in combination therapy with fulvestrant for postmenopausal men and women with human epidermal growth factor receptor 2 (HER2)-negative, PIK3CA-mutated, hormone receptor (HR)-positive, chronic or metastatic breast tumor as diagnosed after progression during or after endocrine-based regimen(22).

We have therefore focused our efforts on a clear understanding of alpelisib's cancer biology to create a comprehensive scientific strategy that can result in the formation of an intervention to counter and/or overcome the acquired resistance to this inhibitor. In the current study, we demonstrate that regulation of AKT/FoxO3a-dependent Bim induction and AKT/GSK-3 $\beta$-dependent Mcl-1 degradation is crucial proceedings that facilitate the potency of alpelisib selective treatment for PIK3CA-activating mutations in breast tumor. We are therefore proposing a robust approach to overcome the resistance of alpelisib through attenuating those events.

\section{Materials And Methods}

\section{Cell culture}

The breast cancer cell lines PIK3CA-mutant including MDA-MB-361 and BT474 were purchased from the American Type Culture Collection (ATCC, VA, USA). The alpelisib-resistant cell lines MDA-BT474-AR and MB-361-AR were established by adding gradually increasing concentrations of alpelisib to BT474 or MDA-MB-361 cells for approximately 6 months. The routine culture of these cell lines was performed in the presence of alpelisib (500 nmol/L), and the cells-maintained resistance to alpelisib following extraction of alpelisib from the medium for 6 or more months, thus showing that an irreversible phenotype was established. All cell lines were cultured in Dulbecco's Modified Eagle Medium (DMEM) with $10 \%$ heat-inactivated $\mathrm{FBS}$ at $37^{\circ} \mathrm{C}$ with $5 \% \mathrm{CO}_{2}$ and $95 \%$ air. Cell lines were consistently evaluated for mycoplasma contamination using MycoAlert Mycoplasma Detection Kit (Sigma, MO, USA), and cell phenotype was analyzed through FACS. Alpelisib, perifosine, and MK-2206 were purchased from Selleckchem (Houston, TX, USA). Cycloheximide (CHX, Sigma) was dissolved in DMSO at $10 \mathrm{mmol} / \mathrm{L}$, 
aliquoted and fewwzed at $-80^{\circ} \mathrm{C}$ for further experimentation. Just before use, appropriate concentrations were prepared from stock solutions with growth medium.

\section{Cell viability assay}

MTT (3-(4,5 dimethylthiazol-2-yl)-2,5-diphenyltetrazolium bromide) assay was performed to analysis the cell viability according to the previously reported protocol $(23,24)$. In brief, the cells were cultured in $96-$ well plates. The serially diluted compounds were added to a suspension of fresh medium and cells. This was performed in triplicate. After 3 days of exposure, cellular viability was determined by using the MTT reagent (Sigma). The MTT-formazan and colored reaction product were dissolved in DMSO for measuring its absorbance at $540 \mathrm{~nm}$ using a POLARStar Omega Reader (BMG Labtech). Three experiments were performed independently with three replicates each, and their geometric mean was calculated to represent the data.

\section{Colony formation assay}

Cells were cultured in 6-well plates, followed by the addition of alpelisib, and then incubated for 24 hours. Colonies were visualized two weeks after planting, and colonies were stained using crystal violet (Sigma) as previously instructed. All experiments were carried out at least twice with three replicates in each experiment.

\section{Apoptosis assay}

Apoptosis within the cells was analyzed through the Annexin V Apoptosis Detection Kit (BD Biosciences). Breast cancer cells, which included attached and floating cells, were collected and stained with FITCAnnexin V/PI. Following cell washing twice with PBS, FACS (fluorescence-activated cell sorting) was used to quantify the intensity of staining.

\section{Real-time PCR}

Total RNA of breast cancer cells or tissues were harvested through RNeasy Plus Mini Kit from Qiagen base on the manufacturer's instructions. The target RNA expression was evaluated using the kits for reverse transcription (RT) and real-time PCR. Reactions for RT were performed in a volume of $20 \mu \mathrm{L}$ through the PrimeScript RT reagent kit (Sigma), kept at $37^{\circ} \mathrm{C}$ for 0.5 hours and then at $85^{\circ} \mathrm{C}$ for $5 \mathrm{~s}$. To carry out real-time PCR, RT product was diluted with reaction buffer (Takara Inc, Dalian, China), and each reaction was performed on an EP Gradient S Eppendorf Mastercycler from Eppendorf (Germany) under the following conditions: $95{ }^{\circ} \mathrm{C}$ for $30 \mathrm{~s} ; 45$ cycles at $95^{\circ} \mathrm{C}$ for $5 \mathrm{~s}$; and $60{ }^{\circ} \mathrm{C}$ for $30 \mathrm{~s}$. GAPDH (glyceraldehyde-3-phosphate dehydrogenase) was used as the internal control to normalize expression as per the method of comparative $2^{-\triangle \Delta \mathrm{Cq}}$. Primers are listed as follows: Mcl-1, Forward: $5^{\prime-}$ CCAAGAAAGCTGCATCGAACCAT-3', Reverse: 5'-CAGCACATTCCTGATGCCACCT-3'; Bim, Forward: 5'GGCCCCTACCTCCCTACA-3', Reverse: 5'-GGGGTTTGTGTTGATTTGTCA-3'; GAPDH, Forward: 5'AGCCACATCGCTCAGACAC-3', Reverse: 5'-GCCCAATACGACCAAATCC-3'. 


\section{Western blotting}

Western blotting was performed by adopting the previously reported methods (25-27). Cells were lysed by lysis buffer (Sigma Aldrich), and total protein was collected from lysates. This was followed by subjecting of $50 \mu \mathrm{g}$ of protein determined through the BCA method (Sigma), to gel electrophoresis (SDSPAGE). Then, it was transferred to polyvinylidene fluoride (PVDF) membranes, followed by blocking with nonfat milk $5 \%$ in $0.5 \%$ TBS-T buffer for $60 \mathrm{~min}$. Next, the membrane was stained with the primary antibody and incubated at $4{ }^{\circ} \mathrm{Co} / \mathrm{n}$. The next morning, the membrane was stained HRP conjugated Secondary antibody and incubated for $60 \mathrm{~min}$ at RT. Primary antibodies used are: cleaved caspase 3 (9661), cleaved caspase 8 (9496), Bim (2933), Mcl-1 (94296), p-Mcl-1 (4579), p-AKT (4060), AKT (9272), Bcl-2 (15071), Bax (5023), HA (2367), GSK-3ß (12456), p-FoxO3a (9465), FoxO3a (2497), E2F1 (3742), Egr-1 (4153), c-Myc (5605), $\beta$-actin (3700, Cell signaling technology).

\section{Knockdown of genes using short hairpin RNA (shRNA) or siRNA}

The knockdown assay was performed as previous studies $(28,29)$. Cell Signaling Technology provided the Bim siRNA and non-silencing control (siRNA duplexes), which were synthesized as mentioned(30). In plates (12-well), cells were transfected with these siRNAs through HiPerFect transfection reagent (Qiagen) as per the manufacture instructions. Lentiviral Bim shRNAs in pLKO.1(Open Biosystems) was used to established Stable Bim knockdown cell lines.

\section{Transfection}

A human full-length Mcl-1 cDNA, amplified from PCR, was transfected into the pCDNA3.1/V5-His plasmid (Invitrogen). Mutations in Mcl-1was generated through QuickChange XL Site-Directed Mutagenesis Kit (Agilent Technologies). Lentiviruses carrying ectopic Mcl-1 were used to establish a stable cell lines BT474/Mcl-1 (S121A/E125A/S159A/T163A; 4A) and MDA-MB-361/Mcl-1 (4A), the cells were selected with zeocin $(500 \mathrm{ng} / \mathrm{mL})$ for 1 week.

\section{ChIP (Chromatin immunoprecipitation) assay}

The Chromatin Immunoprecipitation Assay Kit (EMD Millipore, MA, USA) was used to perform ChIP with the FoxO3a antibody as per the instructions. Analysis of precipitates was performed by PCR using the primers 5'-GCATTTCCTCACAGAGTTGG-3' and 5'-TGCCACCAAAGATCTCTACC-3', and the Bim promoter fragment harboring putative FoxO3a sites was amplified.

\section{Xenograft model}

The in vivo study was carried out as mentioned previously(23). The treatments include alpelisib (20 $\mathrm{mg} / \mathrm{kg} /$ day, oral gavage), perifosine (15 mg/kg/day, oral gavage), alone, vehicle control, or the combination of alpelisib and perifosine. Measurements via caliper were performed to measure tumor volumes and estimated using the formula $V=\left(\right.$ length $\times$ width $\left.^{2}\right) / 2$. The weight of each mouse was taken before euthanization with $\mathrm{CO}_{2}$ asphyxia at the end of treatment. Then, tumors were extracted, weighed 
and then stored in liquid nitrogen for further use. Protein lysis beffer was used to homogenize the tumor tissue aliquots, and Western blotting was subsequently carried out to determine the indicated proteins.

\section{Statistical analysis}

The mean \pm S.D. from three independent experiments (carried out independently, in triplicate) was used to represent the data. Two groups means were compared using Student's $t$-test, and the difference for the multiple groups was compared by applying ANOVA. $p<0.05$ was considered statistically significant.

\section{Results}

\section{Generation of alpelisib-resistant cells in breast cancer cell lines with PIK3CA mutations}

To determine the ability of alpelisib to induce apoptosis in human breast cancer cells, we generated cell lines of alpelisib-resistant (BT474 and MDA-MB-361), which were treated with alpelisib. The alpelisibresistant cell lines (BT474-AR and MDA-MB-361-AR) exhibited enhanced viability in the presence of $1 \mu \mathrm{M}$ alpelisib (Figure 1A and S1A), and the dose-response curve showed a rightward shift (Figure 1B and S1B). Concurrently, cell cycle arrest due to alpelisib was attenuated in the chronically exposed cells; compared to the parental cells these cells has higher cells amount in the S phase (Figure 1C and S1C).

We next treated BT474-AR, MDA-MB-361-AR, and their parental cells with alpelisib for 24 hours and then detected the cleavage of the caspase. Our results showed that, compared to the control treatment, $1 \mu \mathrm{M}$ alpelisib treatment efficiently enhanced the amount of annexin V-positive cells (Figure 1D and S1D). Furthermore, it can also raise the levels of cleaved forms of caspase-3 and caspase-8 (Figure 1E and S1E) in the drug-sensitive parental cells. However, alpelisib treatment didn't rise the annexin V-positive cell percentage or induce the cleavage of caspase-8 and caspase-3 in the alpelisib-resistant cells. In summary, these data suggest that alpelisib treatment prompts apoptosis in drug-sensitive breast cancer cells with mutated PIK3CA.

\section{Alpelisib modulates Bim and Mcl-1 levels and suppresses AKT-dependent Bim induction and Mcl-1 phosphorylation in breast cancer cell lines with mutated PIK3CA}

For understanding alpelisib-mediated apoptotic induction of mutated PIK3CA in drug-sensitive breast cancer cell lines, we investigated whether alpelisib affects the protein level that regulates the Bcl-2 family proteins. Alpelisib treatment with dose of $10-1000 \mathrm{nmol} / \mathrm{L}$ didn't adjust the expression levels of $\mathrm{Bcl}-2$ and Bax in the parental cell lines. However, alpelisib treatment decreased Mcl-1 levels, increased Bim $\mathrm{EL}_{\mathrm{L}}$ expression, and reduced p-AKT levels in these cell lines (Figure 1F and S1F). Within 6 hours Mcl-1 reduced and elevation of Bim elevated. after alpelisib treatment (Figure 1G and S1G). Nevertheless, these alterations didn't occur or minimally occurred in the resistant cell lines, even when they were exposed to 1 $\mu \mathrm{M}$ alpelisib (Figure $1 \mathrm{H}$ and $\mathbf{S 1 H}$ ). Thus, alpelisib modulates $\mathrm{Mcl}-1$ and Bim levels by suppressing AKT in drug-sensitive PIK3CA-mutant breast cancer cell lines. 
Fox03a mediates alpelisib-induced Bim expression in breast cancer cells with mutated PIK3CA

Next, we demonstrate that whether alpelisib affects levels of Mcl-1 and Bim and study the mechanism through which this occurs in drug-sensitive PIK3CA-mutant breast cancer cells. In both BT474 and MDAMB-361 cells, alpelisib treatment substantially showed the induction of Bim mRNA expression compared with the control treatment (Figure 2A and S2A). Next, we determine the mechanism of Bim induction in response to alpelisib. Transcription factors includingE2F1(31), Egr-1(32) and c-Myc(33) are not involved due to unchanged expression following alpelisib treatment (Figure S2B). In a previous study, the induction of Bim could be regulated by the transcription factor FoxO3a in $\mathrm{T}$ and mast cells after cytokine deprivation. Interestingly, we found that siRNA depletion of FoxO3a in BT474 cells abrogated Bim induction by alpelisib, indicating that FoxO3a is needed for Bim induction after kinase inhibition (Figure 2B). Furthermore, the ChIP assay indicated that FoxO3a is directly recruited to the Bim promoter. The binding of FoxO3a to the Bim promoter was enhanced statistically following treatment with alpelisib (Figure 2C). Our findings also indicated that alpelisib prompted dephosphorylation of FoxO3a (Figure 2D), which stalls its consequent transactivation and nuclear translocation. Conversely, active AKT transfection constitutively induced phosphorylation of FoxO3a (Figure 2E), and inhibited Bim induction by alpelisib (Figure 2E). Consequently, AKT inhibition cause FoxO3a-mediated Bim induction following alpelisib treatment.

\section{Alpelisib modulates Mcl-1 levels through AKT/GSK-3ß-dependent degradation in drug-sensitive breast cancer cells with mutated PIK3CA}

Next, we investigated the mechanism by which alpelisib induces Mcl-1 depletion. Our findings indicated that alpelisib did not affect the mRNA level of Mcl-1 (Figure 2F and S2C). We also found speedy degradation of Mcl-1 in alpelisib-treated BT474 or MDA-MB-361 cells than in cells mixed with DMSO (Figure 2G and S2D). Unswervingly, the occurrence of MG132, a proteasome inhibitor, enhanced Mcl-1 basal amount and inhibited the alpelisib-induced reduction in Mcl-1 (Figure $2 \mathrm{H}$ and $\mathrm{S2E}$ ), indicating that the protein degradation depended on the ubiquitin/proteasome system.

In alpelisib-treated cells, phosphorylation of Mcl-1 at Ser159 was observed (Figure 2I). GSK3 $\beta$ phosphorylates $\mathrm{Mcl}-1$ to promote its degradation and is inhibited by AKT through Ser9 phosphorylation. Our results showed that alpelisib prevented Ser9 phosphorylation of GSK3 $\beta$ through analyzing by upstream kinase signaling (Figure S2F). Treating cells with the SB216763(GSK3 $\beta$ inhibitor) inhibits alpelisib-induced phosphorylation and degradation of Mcl-1 (Figure 2J). These data indicate that alpelisib-mediated phosphorylation of Mcl-1 is dependent on AKT/GSK3ß, which facilitates proteasomal degradation.

\section{Modulation of Bim and Mcl-1 levels is an important mechanism responsible for alpelisib-mediated apoptotic induction in drug-sensitive breast cancer cells with mutated PIK3CA}

In order to evaluate whether Bim rise can induce apoptosis by alpelisib, we utilized Bim siRNAs to knockdown Bim expression and then analyzed its effect upon alpelisib's capability to cause apoptosis in 
BT474. Bim siRNA effectively prevented the Bim expression triggered by alpelisib, as found through Western blotting (Figure 3A). Thereafter, we showed decreased amounts of cleaved caspase-3 in transfected cells with siRNA to Bim than that in transfected cells with siRNA control (Figure 3A). Following this finding, we also found considerably less apoptotic cells in BT474 cells transfected with Bim siRNA than in siRNA-transfected cells under regulation (Figure 3B). Consequently, Bim elevation blockade prevents the apoptosis that alpelisib induces. Consistent with our previous results, which reported that MDA-MB-361 cells in which Bim expression using Bim siRNA was knocked down (Figure S3A and S3B).

Next, we study if a reduction in $\mathrm{Mcl}-1$ levels has a role in alpelisib-induced apoptosis in drug-sensitive breast cancer cells with mutated PIK3CA. For this, we expressed ectopic Mcl-1 (S121A/E125A/S159A/T163A; 4A) in both BT474 and MDA-MB-361 cell lines and then checked its effects on alpelisib-induced apoptosis. We found that alpelisib significantly induced caspase cleavage of -3 and -8 (Figure 3 C and S3C) and enhanced the percentage of apoptotic cells (Figure 3D and S3D) in vector-control cell lines. However, it minimally or doesn't affect BT474/Mcl-1 (4A) or MDA-MB-361/Mcl-1 (4A) cells. These findings reveal that the expression of $\mathrm{Mcl}-1$ shields cancer cells from alpelisib-induced apoptosis.

\section{AKT inhibitors restore Bim elevation and Mcl-1 reduction in alpelisib-resistant PIK3CA-mutant breast cancer cell lines}

Present results indicate key roles for $\mathrm{Mcl}-1$ and Bim modulation in controlling alpelisib-mediated apoptotic induction in PIK3CA-mutant breast cancer cell lines. Alpelisib 's inability to simulate these protein levels in BT474-AR and MDA-MB-361-AR cells to acquired alpelisib resistance prompted us to hypothesize whether there is a mechanism that boosts Bim levels with simultaneous Mcl-1 suppression to reduce the threshold for alpelisib-resistant cells to respond to alpelisib, leading to apoptotic cell death. Provided that AKT controls Bim induction and the phosphorylation and degradation of Mcl-1, we considered that AKT suppression contributes to Bim induction and lessening of Mcl-1 in PIK3CA-mutant alpelisib-resistant breast cancer cells. In the alpelisib-resistant cell lines, BT474-AR and MDA-MB-361-AR, alpelisib was not able to suppress AKT, increase Bim levels, and reduced Mcl-1 levels (Figure 4A-4D). These finding additionally verify the significance of Bim levels in defining cell sensitivity to alpelisib and subduing AKT signaling and modulating Mcl-1. Two different AKT inhibitors, MK-2206 and perifosine, individually or in combination with alpelisib, effectively suppressed AKT phosphorylation, increased Bim expression, and reduced Mcl-1 expression in the mentioned resistant cell lines (Figure 4A-4D). Our results confirm that AKT inhibitors suppress AKT phosphorylation, reduce $\mathrm{Mcl}-1$, and increase Bim in alpelisibresistant breast cancer cell lines with mutated PIK3CA.

\section{AKT inhibitors effectively overcome the acquired resistance of PIK3CAmutant breast cancer cells to alpelisib}

Next, we evaluate if AKT inhibition indeed restored the sensitivity of alpelisib-resistant breast cancer cells to alpelisib. MK-2206 and perifosine (AKT inhibitors) at the determined concentration ranges didn't 
suppress the growth of BT474-AR cells, nor every week. As envisioned, this cell line was alpelisibresistant. The combined effect of alpelisib for each of the AKT inhibitors however very efficiently prevents the development of BT474-AR cells (Figure 5A-5D and S4A-S4D). The Cls were well below 0.5, suggesting highly synergistic growth inhibitory effects. In long-term colony formation testing, we observed that the combining alpelisib with either of the two AKT inhibitors abolished colony formation, while either agent itself inhibited the development of BT474-AR cells in colony mildly. (Figure 5E-5F and S4E-S4H). Taken together, these data suggest that AKT inhibitors restore the sensitivity of alpelisib-resistant cells to alpelisib to efficiently stunned alpelisib resistance.

\section{Alpelisib in combination with an AKT inhibitor effectively induces the apoptosis of alpelisib-resistant breast cancer cells}

Additionally, we evaluated the effects of alpelisib in combination with an AKT inhibitor on apoptotic induction in alpelisib-resistant breast cancer cells. Alpelisib or perifosine alone didn't increase or tend to enhance the percentage of apoptotic cells (Figure 5G) and the cleavage of caspase-8 and caspase-3 (Figure 5H) in BT474-AR and MDA-MB-361-AR cells. Nevertheless, their combination improved the percentage of apoptotic cells (Figure $\mathbf{5 G}$ ) and the cleavage of caspase-8 and caspase-3 (Figure $5 \mathrm{H}$ ). The same results were observed with MK-2206 treatment (Figure 5I-5J). Taken together, these data show that a combination of alpelisib with an AKT inhibitor effectively induces apoptosis in alpelisib-resistant breast cancer cells.

\section{Alpelisib in combination with perifosine effectively inhibits the growth of alpelisib-resistant breast cancer xenografts in a mouse model}

After the promising therapeutic activity of alpelisib in combination with the AKT inhibitor in alpelisibresistant breast cancer cells, next, we evaluate its efficiency in xenograft tumor mice model alpelisibresistant. As shown in vitro, both alpelisib and perifosine alone had no impact or marginal effects on the development of BT474-AR tumors while treated regularly for approximately 3 weeks. On the other hand, when they were combined, they markedly eliminated the growth of these xenografts under the conditions tested (Figure 6A and 6B) without any toxicity determined from the mean body weight observed at an indicted time interval (Figure 6C). We have repeated the MDA-MB-361-AR xenograft experiment with an intermittent treatment schedule of 1 week on treatment, 1 week off treatment, 1 week on treatment. Similar results for possible increased toxicity were observed as shown in Figure S6A-S6C. It is, therefore, obvious that the combination therapy can successfully inhibit the growth of alpelisib-resistant tumors in vivo with no obvious toxicity.

In xenograft tumors of mice undergoing the specified therapies, we further measured p-AKT, Mcl-1, and Bim rates. In consistence with in vitro observations, perifosine alone significantly raises the levels of Bim and decrease levels of Mcl-1 and p-AKT, while alpelisib alone didn't alter concentrations of the proteins. The combination of alpelisib and perifosine was not extra effective than perifosine alone at rising Bim levels but significantly reduced Mcl-1 levels beyond the level observed with the single agent (Figure 6D). Furthermore, the alpelisib and perifosine combination induced more apoptosis in BT474-AR tumors than 
single agents alone (Figure 6E). Therefore, we found that in vivo regulation of $\mathrm{Mcl}-1$ and Bim rates by the combination of alpelisib and the AKT inhibitor.

\section{Discussion}

In breast cancer, the most commonly mutated oncogene observed is PIK3CA, which sustains activating mutations at a high frequency in many other types of cancer $(34,35)$. Therefore, multiple clinical trials are being carried out to evaluate small-molecule PI3K inhibitors, along with other anticancer drugs(36-38). Nevertheless, their clinical advantages are marred by acquired and intrinsic resistance to PI3K inhibitors(38). Elucidating how cancer cells overcome PI3K inhibition may expedite approaches to develop new therapeutics for breast cancer with mutated PIK3CA and various tumors dependent on PI3K.

The present study demonstrates that alpelisib efficiently induces apoptosis in the breast cancer cell with mutated PIK3CA but this doesn't occur in alpelisib-insensitive breast cancer cell lines that have acquired resistance to alpelisib. The induction of apoptosis is a crucial step for the alpelisib's therapeutic effectiveness for PIK3CA-mutant breast cancers. Our data also demonstrate that alpelisib enhance Bim levels and decrease Mcl-1 levels in drug-sensitive PIK3CA-mutant breast cancer cells, although not in alpelisib-resistant cells, including BT474-AR and MDA-MB-361-AR, strongly suggesting that the modulation of both Bim and Mcl-1 levels is crucial for mediating alpelisib-induced apoptosis. This was corroborated with our observations that both, the inhibition of Bim induction or the enforced expression of an Mcl-1 ectopic mutant in the drug-sensitive PIK3CA-mutant breast cancer cells successfully protects them from alpelisib-induced apoptosis.

As a member of the Bcl-2 family, Bim can be activated through various pro-apoptotic stimuli and facilitate cell death that is dependent on Bak and Bax(39). The FOXO (Forkhead box 0) transcription factors participate in several biochemical processes, such as cell-cycle arrest, apoptosis, cell metabolism, and DNA damage repair(40). Several external stimuli, such as insulin, IGF-1, and a few other growth factors, can modulate FOXO transcription factors(41). The FOXO member that is the most highly expressed, Foxo3a, correlates with cancer progression(42). In our study, we found that the pro-apoptotic function of Bim induced by alpelisib depends on Foxo3a.

The Mcl-1 protein has a short half-life and consists of PEST (proline-, glutamic acid-, serine- and threonine-rich) sequences, which have a crucial role in the degradation of proteins $(43,44)$. Therefore, proteasome-mediated degradation tightly regulates the expression of $\mathrm{Mcl}-1$ (45). Four sites of phosphorylation, including S121, E125, S159 and T163, have been identified on Mcl-1(46-47). These sites can be phosphorylated by several kinases to facilitate the degradation of $\mathrm{Mcl}-1$ in response to numerous stimuli(48-50). In this study, alpelisib had no noticeable effect on the expression of Mcl-1 mRNA, and the alpelisib-induced reduction of Mcl-1 was markedly blocked by MG132. Furthermore, the extent of ubiquitination of Mcl-1 was significantly increased in the presence of alpelisib. Moreover, GSK-3 $\beta$ is involved in alpelisib-induced Mcl-1 degradation(45). Thus, alpelisib downregulates Mcl-1 by a process closely related to proteasomal degradation. 
Our research has identified a critical mechanism: the necessary outcome of reduction of Mcl-1 in PIK3CAmutant breast cancer cells mediating alpelisib-induced apoptosis. Thus, we conclude that alpelisib enhances Bim levels by promoting AKT/FoxO3a-dependent Bim induction. Also, we found that alpelisib reduced the levels of Mcl-1 and enhanced the phosphorylation of Mcl-1 at S159. Moreover, alpelisib increases the rate of Mcl-1 degradation, and proteasome inhibition with MG132 attenuated the decline in $\mathrm{Mcl}-1$ induced by alpelisib. Therefore, we recommend that alpelisib reduces $\mathrm{Mcl}-1$ levels through enhanced AKT/GSK-3ß-dependent degradation. These data that numerous AKT inhibitors successfully rise Bim levels and decrease Mcl-1 levels and suppress AKT in alpelisib-resistant PIK3CA mutant breast cancer cells also support this model of modulation of Bim induction and Mcl-1 degradation. Thus, our findings have introduced a unique mechanism in this respect by which alpelisib modulates the levels of Mcl-1 and Bim, leading to apoptotic induction. Consequently, alpelisib 's failure to modify Mcl-1 and Bim rates by altering AKT-dependent Bim induction and Mcl-1 reduction in alpelisib-resistant cells constitutes a strategic mechanism whereby the drug-sensitive PIK3CA-mutant breast cancer cells may become immune to apoptosis induction by alpelisib.

We do not currently know why the AKT signaling pathway in alpelisib-resistant cell lines passive to the treatment with alpelisib, but we plan to address this issue in the near future. However, we reported a therapeutic approach established on the concept that alpelisib-resistant cells can overcome resistance by co-targeting AKT signaling. Through inducing Bim and growing Mcl-1 rates through suppressing AKTdependent Bim induction and degrading Mcl-1 with an AKT inhibitor, we have restored the vulnerability of alpelisib-resistant breast cancer cells with mutated PIK3CA to apoptotic death, exhibiting encouraging anticancer results toward alpelisib-resistant breast cancer cell growth, both in vivo and in vitro. By attacking AKT signaling, we obtained similar findings with 2 separate AKT inhibitors, thereby illustrating the reason for overcoming alpelisib resistance. Nonetheless, increased induction of Bim and reduction of Mcl-1 levels will result in an increased ratio of Bim to $\mathrm{Mcl}-1$ (Bim: $\mathrm{Mcl}-1)$ in the present resistant cell line, ultimately increasing apoptotic induction and suppression of tumor growth.

\section{Conclusion}

To sum up, this study has reported a vital function of Bim and Mcl-1 modulation in carrying out apoptotic induction in response to alpelisib in drug-sensitive breast cancer cell lines with mutated PIK3CA. Based on this significant observation, we propose that a successful therapeutic strategy to address acquired resistance to alpelisib would be co-targeting AKT signaling.

\section{Abbreviations}

ATCC: American Type Culture Collection; ChIP: Chromatin immunoprecipitation; CHX: Cycloheximide; DMEM: Dulbecco's Modified Eagle Medium; ER: estrogen receptor; FACS: fluorescence-activated cell sorting; FOXO: Forkhead box 0; GAPDH: glyceraldehyde-3-phosphate dehydrogenase; GPCRs: G proteincoupled receptors; HER2: human epidermal growth factor receptor 2; HR: hormone receptor; HRP: horseradish peroxidase; MTT: 3-(4,5 dimethylthiazol-2-yl)-2,5-diphenyltetrazolium bromide; PH: pleckstrin 
homology; PI(4,5)P2: phosphatidylinositol (4,5)-bisphosphate; PI3Ka: phosphatidylinositol 3-kinase a; PIP3: phosphatidylinositol $(3,4,5)$-trisphosphate; PVDF: polyvinylidene fluoride; RT: reverse transcription; RTKs: receptor tyrosine kinases.

\section{Declarations}

\section{Ethics approval and consent to participate}

This study was approved by the Ethical Committee of the Xiangya Hospital, Central South University.

\section{Consent for publication}

Informed consent was obtained from all individual participants included in the study.

\section{Availability of data and materials}

All data generated or analyzed during this study are included in this published article.

\section{Competing interests}

The authors declare that they have no competing interests.

\section{Funding}

No applicable

\section{Authors' contributions}

X.T. and JS.T. developed the hypothesis and designed the experiments. X.T., Z.Z., P.L. and H.L. performed the statistical analyses. X.T., H.Y. and JS.T. conducted experiments and wrote the main manuscript. All authors read and approved the final manuscript.

\section{Acknowledgments}

No applicable

\section{References}

1. Janku F, Yap TA, \& Meric-Bernstam F (2018) Targeting the PI3K pathway in cancer: are we making headway? Nat Rev Clin Oncol 15(5):273-291.

2. Bilanges $B, P o s o r Y, \&$ Vanhaesebroeck B (2019) PI3K isoforms in cell signalling and vesicle trafficking. Nat Rev Mol Cell Biol 20(9):515-534.

3. Qin H, et al. (2018) The impact of PI3K inhibitors on breast cancer cell and its tumor microenvironment. PeerJ 6:e5092. 
4. Luo J, Manning BD, \& Cantley LC (2003) Targeting the PI3K-Akt pathway in human cancer: rationale and promise. Cancer Cell 4(4):257-262.

5. Liu P, Cheng H, Roberts TM, \& Zhao JJ (2009) Targeting the phosphoinositide 3-kinase pathway in cancer. Nat Rev Drug Discov 8(8):627-644.

6. Liu D, Hou P, Liu Z, Wu G, \& Xing M (2009) Genetic alterations in the phosphoinositide 3-kinase/Akt signaling pathway confer sensitivity of thyroid cancer cells to therapeutic targeting of Akt and mammalian target of rapamycin. Cancer Res 69(18):7311-7319.

7. Dienstmann R, Rodon J, Serra V, \& Tabernero J (2014) Picking the point of inhibition: a comparative review of PI3K/AKT/mTOR pathway inhibitors. Mol Cancer Ther 13(5):1021-1031.

8. van de Stolpe A (2019) Quantitative Measurement of Functional Activity of the PI3K Signaling Pathway in Cancer. Cancers (Basel) 11(3).

9. Dbouk HA, et al. (2012) G protein-coupled receptor-mediated activation of p110beta by Gbetagamma is required for cellular transformation and invasiveness. Sci Signal 5(253):ra89.

10. Lien EC, Dibble CC, \& Toker A (2017) PI3K signaling in cancer: beyond AKT. Curr Opin Cell Biol 45:6271.

11. Miao B, et al. (2010) Small molecule inhibition of phosphatidylinositol-3,4,5-triphosphate (PIP3) binding to pleckstrin homology domains. Proc Natl Acad Sci U S A 107(46):20126-20131.

12. Manning BD \& Toker A (2017) AKT/PKB Signaling: Navigating the Network. Cel/ 169(3):381-405.

13. Janku $F(2017)$ Phosphoinositide 3-kinase (PI3K) pathway inhibitors in solid tumors: From laboratory to patients. Cancer Treat Rev 59:93-101.

14. Curigliano G \& Shah RR (2019) Safety and Tolerability of Phosphatidylinositol-3-Kinase (PI3K) Inhibitors in Oncology. Drug Saf 42(2):247-262.

15. Asati V, Bharti SK, Mahapatra DK, Asati V, \& Budhwani AK (2016) Triggering PIK3CA Mutations in $\mathrm{PI3K} / \mathrm{Akt} / \mathrm{mTOR}$ Axis: Exploration of Newer Inhibitors and Rational Preventive Strategies. Curr Pharm Des 22(39):6039-6054.

16. Liu S, Tang Y, Yan M, \& Jiang W (2018) PIK3CA mutation sensitizes breast cancer cells to synergistic therapy of PI3K inhibition and AMPK activation. Invest New Drugs 36(5):763-772.

17. Ravegnini G, et al. (2019) Mechanisms of resistance to a PI3K inhibitor in gastrointestinal stromal tumors: an omic approach to identify novel druggable targets. Cancer Manag Res 11:6229-6244.

18. Toska E, et al. (2017) PI3K pathway regulates ER-dependent transcription in breast cancer through the epigenetic regulator KMT2D. Science 355(6331):1324-1330.

19. Chen IC, et al. (2017) Phosphatidylinositol-3 Kinase Inhibitors, Buparlisib and Alpelisib, Sensitize Estrogen Receptor-positive Breast Cancer Cells to Tamoxifen. Sci Rep 7(1):9842.

20. Keegan NM, Gleeson JP, Hennessy BT, \& Morris PG (2018) PI3K inhibition to overcome endocrine resistance in breast cancer. Expert Opin Investig Drugs 27(1):1-15.

21. Okazaki M, et al. (2018) Predictive markers for efficacy of everolimus plus exemestane in patients with luminal HER2-negative metastatic breast cancer. Med Onco/35(4):48. 
22. Stirrups R (2019) Alpelisib plus fulvestrant for PIK3CA-mutated breast cancer. Lancet Oncol 20(7):e347.

23. Zhang Z, et al. (2018) GNA13 promotes tumor growth and angiogenesis by upregulating CXC chemokines via the NF-kappaB signaling pathway in colorectal cancer cells. Cancer Med 7(11):56115620 .

24. Jiang B, et al. (2020) Down-regulation of long non-coding RNA HOTAIR promotes angiogenesis via regulating miR-126/SCEL pathways in burn wound healing. Cell Death Dis 11(1):61.

25. Tan X, Zhang Z, Yao H, \& Shen L (2018) Tim-4 promotes the growth of colorectal cancer by activating angiogenesis and recruiting tumor-associated macrophages via the PI3K/AKT/mTOR signaling pathway. Cancer Lett 436:119-128.

26. Qiu L, Zhou G, \& Cao S (2020) Targeted inhibition of ULK1 enhances daunorubicin sensitivity in acute myeloid leukemia. Life Sci 243:117234.

27. Deng JL, Zhang R, Zeng Y, Zhu YS, \& Wang G (2019) Statins induce cell apoptosis through a modulation of AKT/FOXO1 pathway in prostate cancer cells. Cancer Manag Res 11:7231-7242.

28. Xu Y, et al. (2019) The LRP6 functional mutation rs2302685 contributes to individual susceptibility to alcoholic liver injury related to the Wnt/beta-catenin-TCF1-CYP2E1 signaling pathway. Arch Toxico/93(6):1679-1695.

29. Ding NH, et al. (2018) NEK4 kinase regulates EMT to promote lung cancer metastasis. J Cell Mol Med 22(12):5877-5887.

30. Tong JS, et al. (2010) ER-alpha36, a novel variant of ER-alpha, mediates estrogen-stimulated proliferation of endometrial carcinoma cells via the PKCdelta/ERK pathway. PLoS One 5(11):e15408.

31. Wu Y, et al. (2017) Loss of GCN5 leads to increased neuronal apoptosis by upregulating E2F1- and Egr-1-dependent BH3-only protein Bim. Cell Death Dis 8(1):e2570.

32. Xie B, et al. (2011) Egr-1 transactivates Bim gene expression to promote neuronal apoptosis. $J$ Neurosci 31(13):5032-5044.

33. Campone M, et al. (2011) c-Myc dependent expression of pro-apoptotic Bim renders HER2overexpressing breast cancer cells dependent on anti-apoptotic Mcl-1. Mol Cancer 10:110.

34. Zardavas D, Phillips WA, \& Loi S (2014) PIK3CA mutations in breast cancer: reconciling findings from preclinical and clinical data. Breast Cancer Res 16(1):201.

35. Pang B, et al. (2014) Prognostic role of PIK3CA mutations and their association with hormone receptor expression in breast cancer: a meta-analysis. Sci Rep 4:6255.

36. Lehmann BD, et al. (2019) TBCRC 032 IB/II Multicenter Study: Molecular insights to AR antagonist and $\mathrm{PI3K}$ inhibitor efficacy in patients with $\mathrm{AR}+$ metastatic triple-negative breast cancer. Clin Cancer Res.

37. Ippen FM, et al. (2019) The Dual PI3K/mTOR Pathway Inhibitor GDC-0084 Achieves Antitumor Activity in PIK3CA-Mutant Breast Cancer Brain Metastases. Clin Cancer Res 25(11):3374-3383. 
38. Jain S, et al. (2018) Phase I study of alpelisib (BYL-719) and trastuzumab emtansine (T-DM1) in HER2-positive metastatic breast cancer (MBC) after trastuzumab and taxane therapy. Breast Cancer Res Treat 171(2):371-381.

39. Kale J, Osterlund EJ, \& Andrews DW (2018) BCL-2 family proteins: changing partners in the dance towards death. Cell Death Differ 25(1):65-80.

40. Tia N, et al. (2018) Role of Forkhead Box O (FOXO) transcription factor in aging and diseases. Gene 648:97-105.

41. Yang S, et al. (2011) Foxo1 mediates insulin-like growth factor 1 (IGF1)/insulin regulation of osteocalcin expression by antagonizing Runx2 in osteoblasts. J Biol Chem 286(21):19149-19158.

42. Farhan M, et al. (2017) FOXO Signaling Pathways as Therapeutic Targets in Cancer. Int J Biol Sci 13(7):815-827.

43. Xiang W, Yang CY, \& Bai L (2018) MCL-1 inhibition in cancer treatment. Onco Targets Ther 11:73017314.

44. Young Al, Timpson P, Gallego-Ortega D, Ormandy CJ, \& Oakes SR (2018) Myeloid cell leukemia 1 (MCL-1), an unexpected modulator of protein kinase signaling during invasion. Cell Adh Migr 12(6):513-523.

45. Inuzuka H, et al. (2011) SCF(FBW7) regulates cellular apoptosis by targeting MCL1 for ubiquitylation and destruction. Nature 471(7336):104-109.

46. Zhang S, et al. (2018) Deubiquitinase USP13 dictates MCL1 stability and sensitivity to BH3 mimetic inhibitors. Nat Commun 9(1):215.

47. Wertz IE, et al. (2011) Sensitivity to antitubulin chemotherapeutics is regulated by MCL1 and FBW7. Nature 471(7336):110-114.

48. Tong J, et al. (2018) Mcl-1 Phosphorylation without Degradation Mediates Sensitivity to HDAC Inhibitors by Liberating BH3-Only Proteins. Cancer Res 78(16):4704-4715.

49. Tong J, et al. (2017) FBW7-Dependent Mcl-1 Degradation Mediates the Anticancer Effect of Hsp90 Inhibitors. Mol Cancer Ther 16(9):1979-1988.

50. Tong J, Tan S, Zou F, Yu J, \& Zhang L (2017) FBW7 mutations mediate resistance of colorectal cancer to targeted therapies by blocking Mcl-1 degradation. Oncogene 36(6):787-796.

\section{Figures}


A

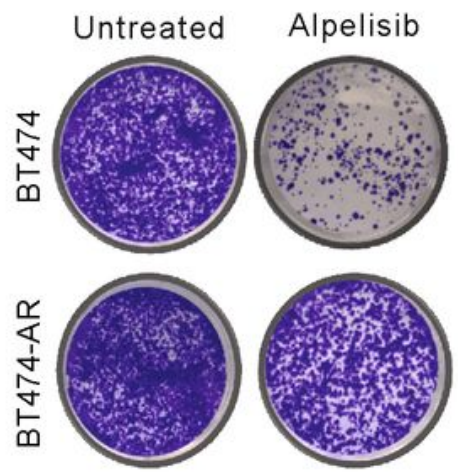

C

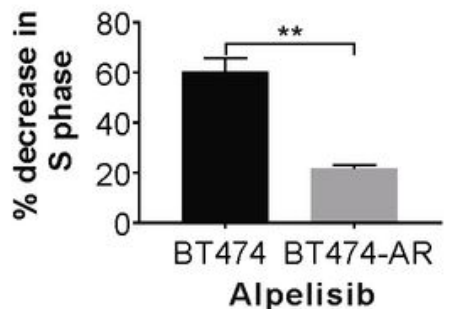

E

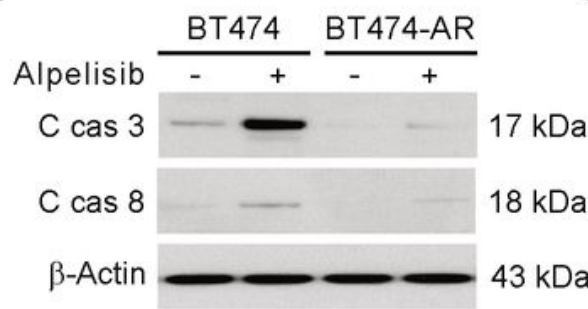

F

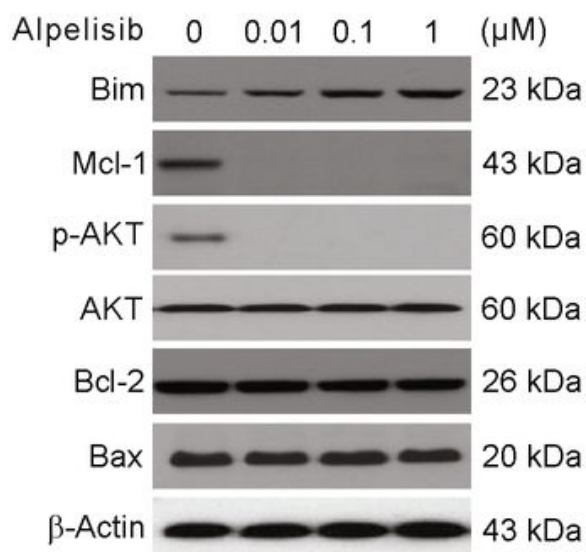

BT474
B

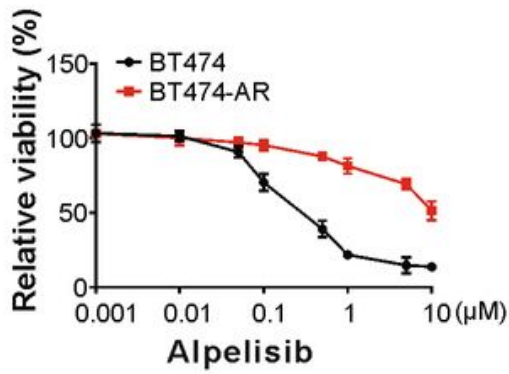

D

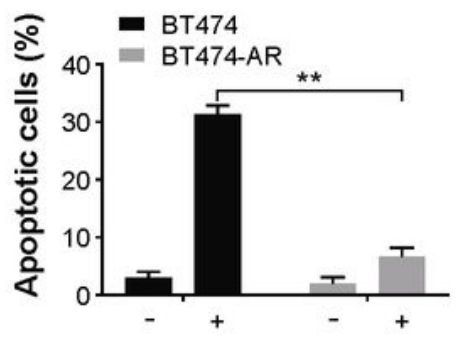

Alpelisib

G

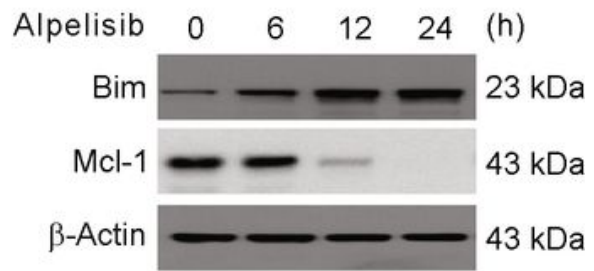

BT474

$\mathrm{H}$

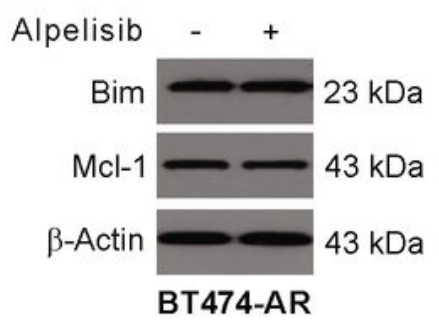

\section{Figure 1}

Acquired resistance to alpelisib breast cancer cell lines generation. (A) Parental and resistant BT474 cells were treated with either the vehicle or $1 \mu \mathrm{M}$ alpelisib for 48 hours. Representative plates are shown. (B) Cells were treated with increasing doses of alpelisib for 72 hours. Viability was assessed using MTT. (C) Parental and resistant BT474 cell lines were treated with alpelisib for 24 hours, after which cell-cycle analysis was performed using propidium iodide staining followed by flow cytometry. The percentage 
decrease in S phase relative to vehicle controls is depicted. (D) Parental and resistant BT474 cells were treated with either the vehicle or $1 \mu \mathrm{M}$ alpelisib for 48 hours. Apoptosis was analyzed by flow cytometry. (E) Parental and resistant BT474 cells were treated with either the vehicle or $1 \mu \mathrm{M}$ alpelisib for 48 hours. Cleaved caspase 3 and 8 were analyzed by Western blotting. (F) Parental and resistant BT474 cells were treated with alpelisib at indicated concentration for 48 hours. Indicated proteins were analyzed by Western blotting. (G) Parental and resistant BT474 cells were treated with $1 \mu \mathrm{M}$ alpelisib at indicated time points. Indicated proteins were analyzed by Western blotting. $(\mathrm{H})$ Indicated cells were treated with $1 \mu \mathrm{M}$ alpelisib for 48 hours. Indicated proteins were analyzed by Western blotting. The results were expressed as the means \pm SD of three independent experiments. ${ }^{*}, \mathrm{P}<0.01$.

A

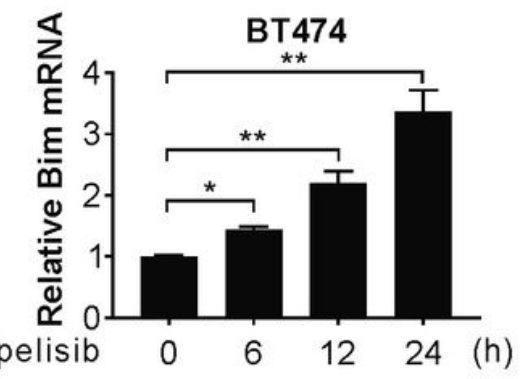

D

G

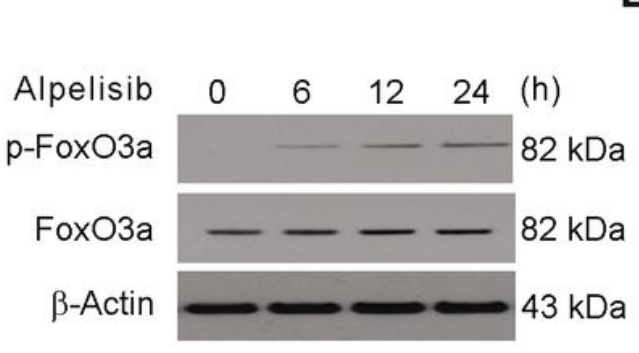

E
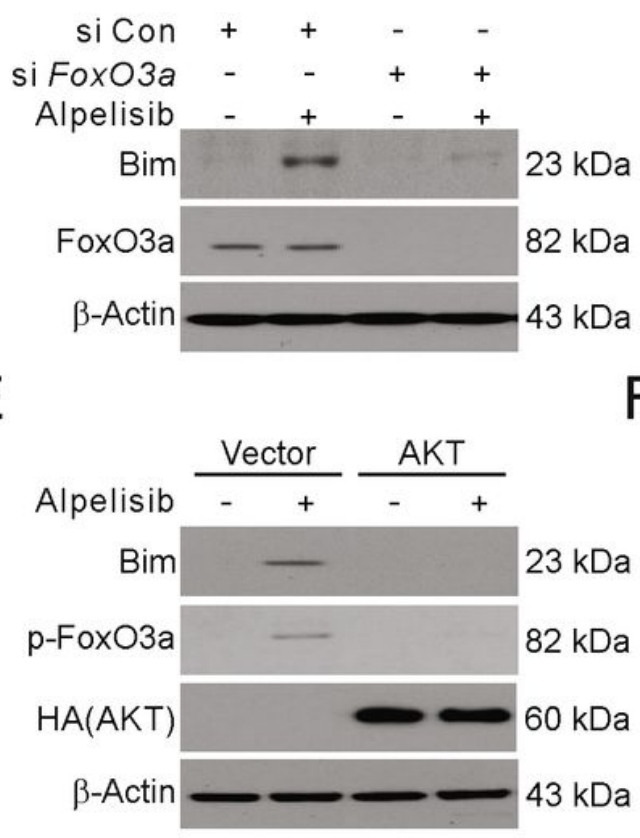

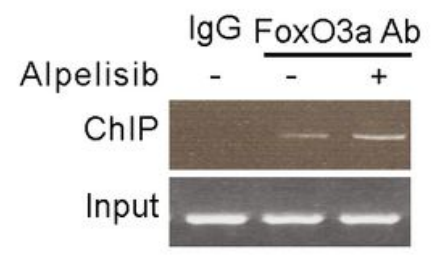

F

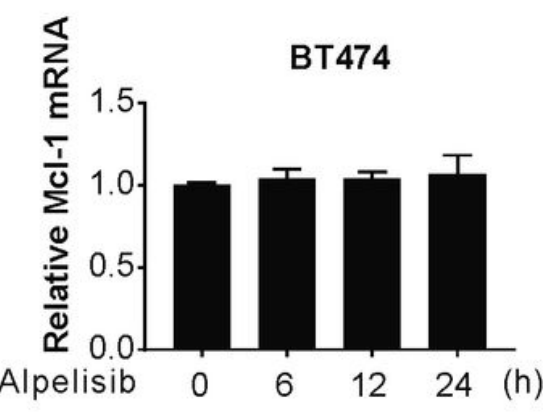

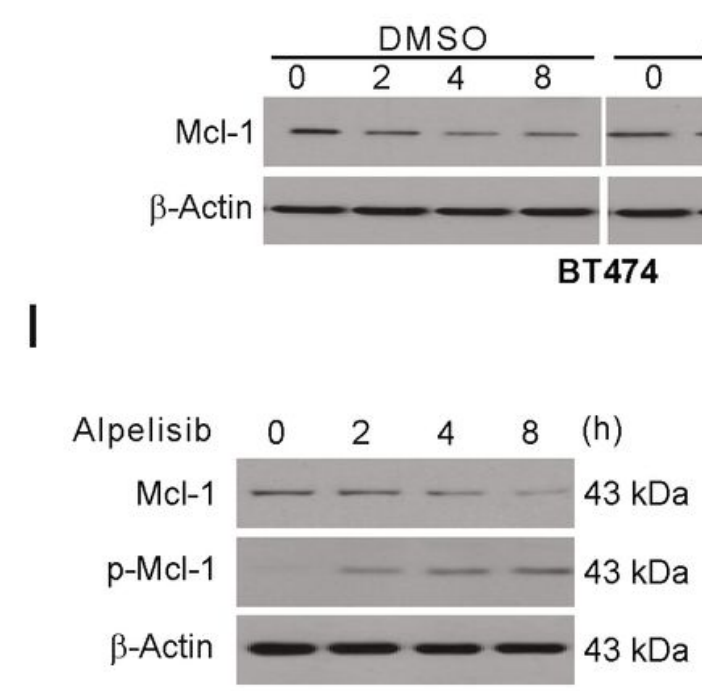

$\mathrm{H}$
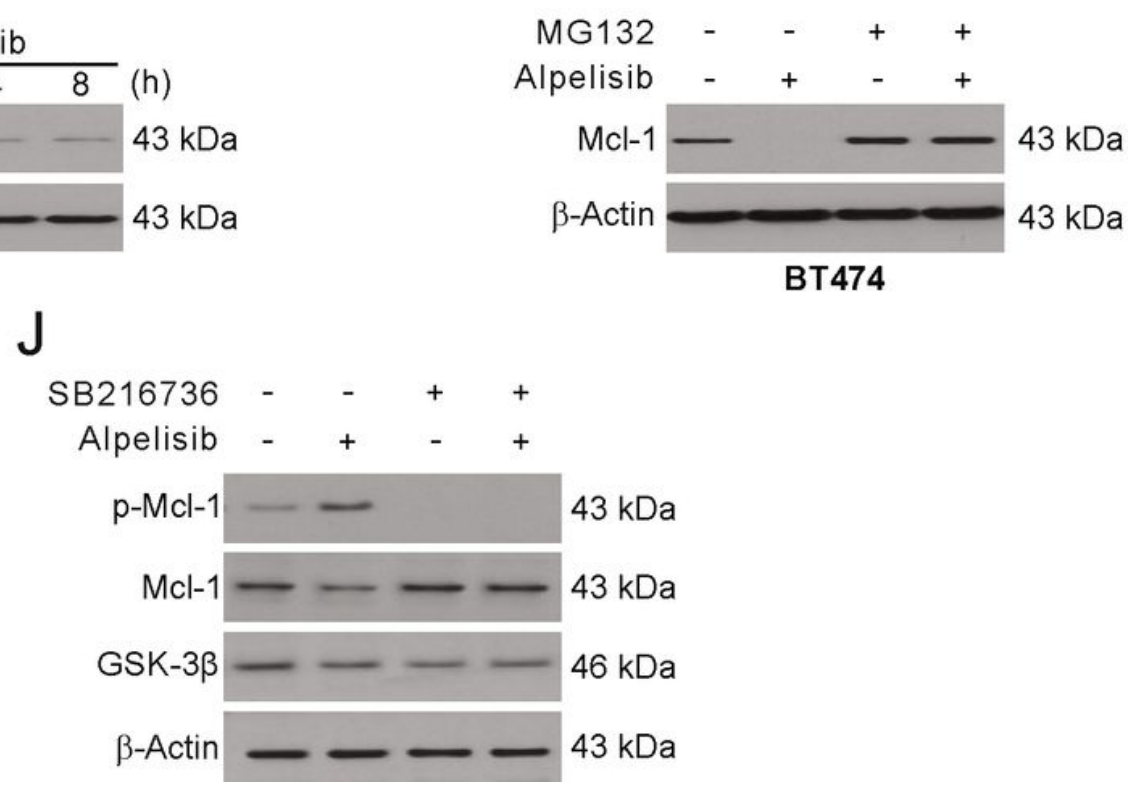

Figure 2 
FoxO3a mediated alpelisib-induced Bim expression and $\mathrm{Mcl}-1$ degradation in breast cancer cells with mutated PIK3CA. (A) BT474 cells were treated with $1 \mu \mathrm{M}$ alpelisib at indicated time points. Bim mRNA level was analyzed by real-time PCR. (B) BT474 cells transfected with Fox03a siRNA were treated with $1 \mu \mathrm{M}$ alpelisib for 48 hours. Indicated proteins were analyzed by Western blotting. (C) Chromatin immunoprecipitation (ChIP) was performed using anti-FoxO3a antibody on BT474 cells following alpelisib $(1 \mu \mathrm{M})$ treatment for 8 hours. The IgG was used to control for antibody specificity. PCR was carried out using primers surrounding the FoxO3a binding sites in the Bim promoter. (D) BT474 cells were treated with $1 \mu \mathrm{M}$ alpelisib at indicated time points. Indicated proteins were analyzed by Western blotting. (E) BT474 cells transfected with constitutively active AKT plasmid were treated with $1 \mu \mathrm{M}$ alpelisib at indicated time points. Indicated proteins were analyzed by Western blotting. (F) BT474 cells were treated with $1 \mu \mathrm{M}$ alpelisib at indicated time points. Relative mRNA level of Mcl-1 was analyzed by real-time PCR. (G) Indicated cell lines were exposed to $1 \mu \mathrm{M}$ alpelisib for 2 hours followed with the addition of $10 \mathrm{mg} / \mathrm{mL}$ $\mathrm{CHX}$. The cells were then harvested at the indicated times for Western blotting to detect the indicated proteins. $(H)$ The indicated cell lines were pretreated with $5 \mathrm{mg} / \mathrm{mL}$ MG132 for 30 minutes and then cotreated with $1 \mu \mathrm{M}$ alpelisib for an additional 4 hours. The indicated protein level was analyzed by Western blotting. (I) BT474 cells were treated with $1 \mu \mathrm{M}$ alpelisib at indicated time points. The indicated protein level was analyzed by Western blotting. (J) BT474 cells were pretreated with $5 \mathrm{mg} / \mathrm{mL}$ SB216736 for 30 minutes and then cotreated with $1 \mu \mathrm{M}$ alpelisib for an additional 4 hours. The indicated protein level was analyzed by Western blotting. The results were expressed as the means \pm SD of three independent experiments. *, $\mathrm{P}<0.05 ; * \star, \mathrm{P}<0.01$. 
A

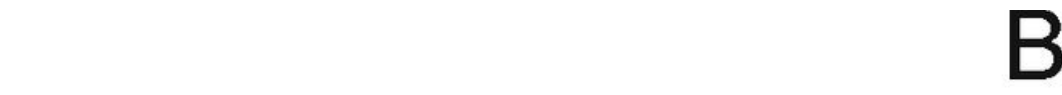

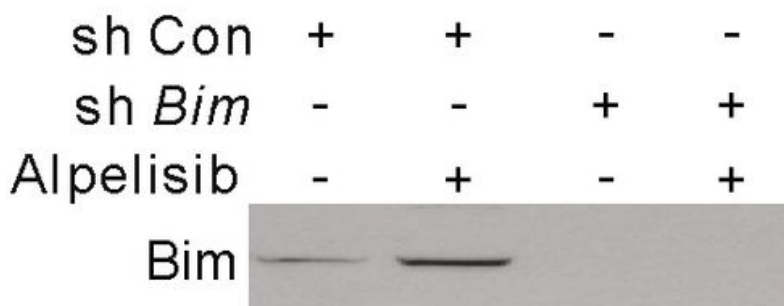

C cas 3

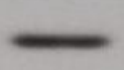

$\beta$-Actin

\section{BT474}

C

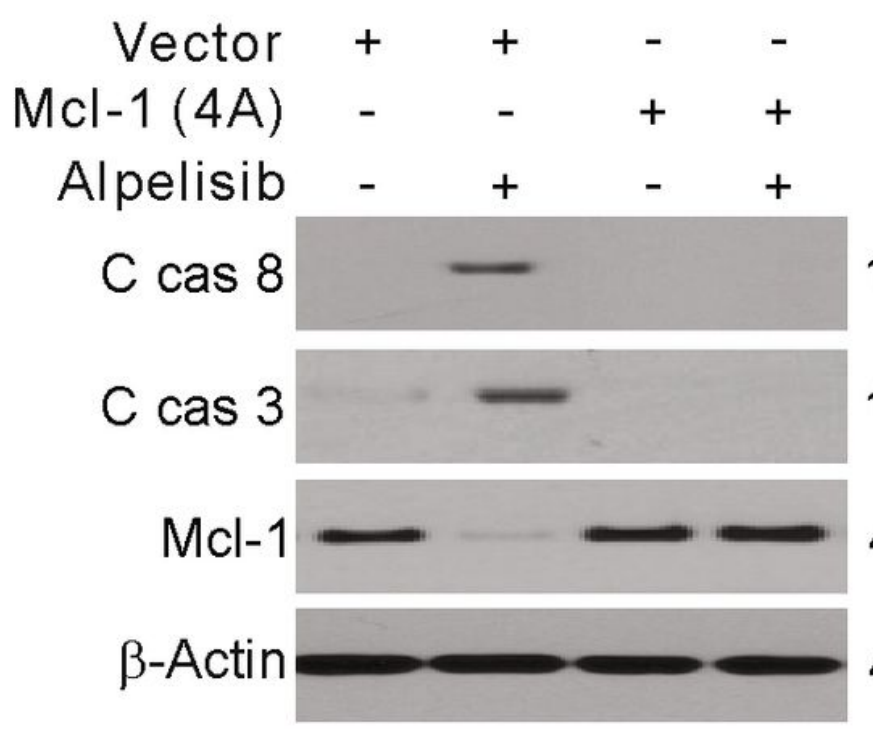

$18 \mathrm{kDa}$

$17 \mathrm{kDa}$

$43 \mathrm{kDa}$

$43 \mathrm{kDa}$

\section{BT474}

$43 \mathrm{kDa}$

$23 \mathrm{kDa}$

$17 \mathrm{kDa}$

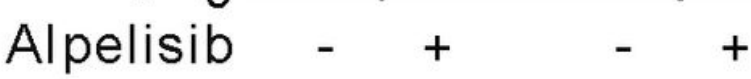

\section{BT474}

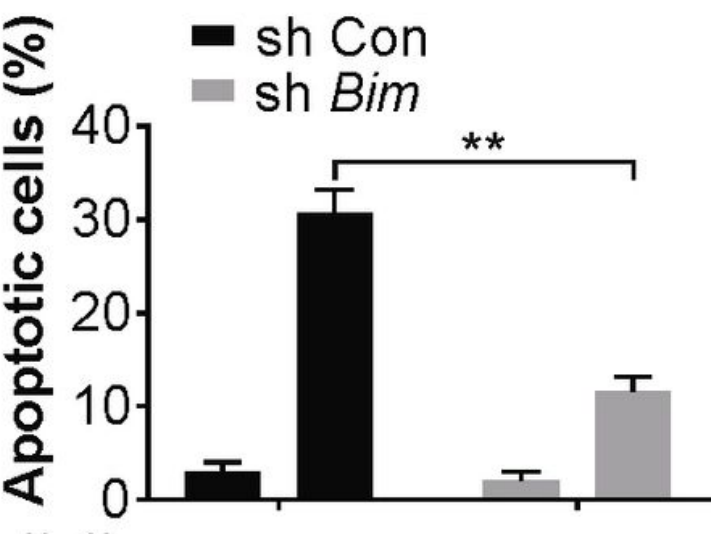

D

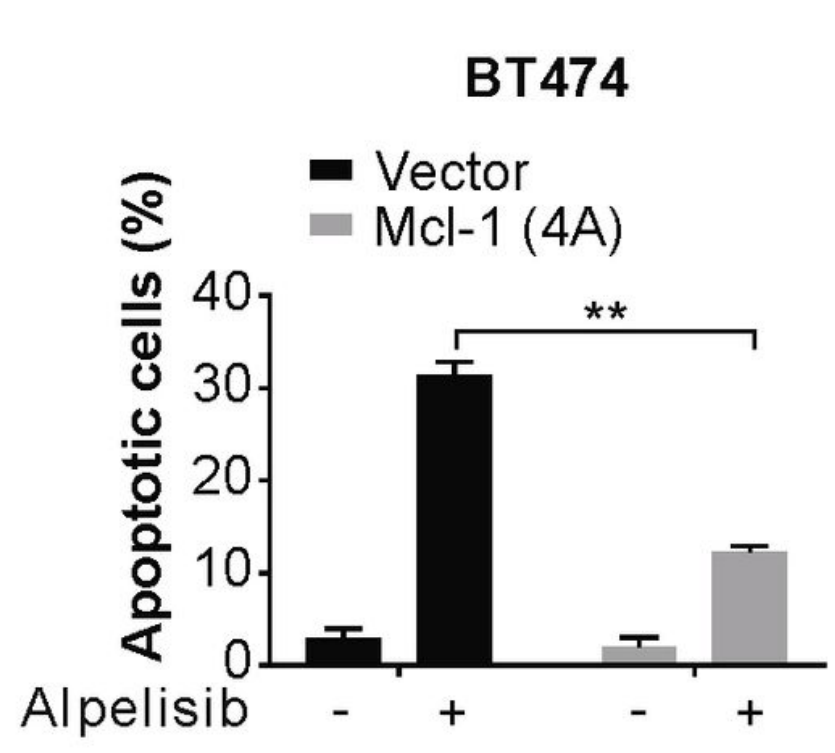

\section{Figure 3}

Bim and $\mathrm{Mcl}-1$ regulate PIK3CA-mutation breast cancer sensitivity to alpelisib. (A) Indicated cells transfected with sh Bim were treated with $1 \mu \mathrm{M}$ alpelisib for 48 hours. The indicated protein level was analyzed by Western blotting. (B) Indicated cells transfected with sh Bim were treated with $1 \mu \mathrm{M}$ alpelisib for 48 hours. Apoptosis was analyzed by flow cytometry. (C) Indicated cells transfected with vector or Mcl-1 (4A) were treated with $1 \mu \mathrm{M}$ alpelisib for 48 hours. The indicated protein level was analyzed by Western blotting. (D) Indicated cells transfected with vector or Mcl-1 (4A) were treated with $1 \mu \mathrm{M}$ alpelisib for 48 hours. Apoptosis was analyzed by flow cytometry. 

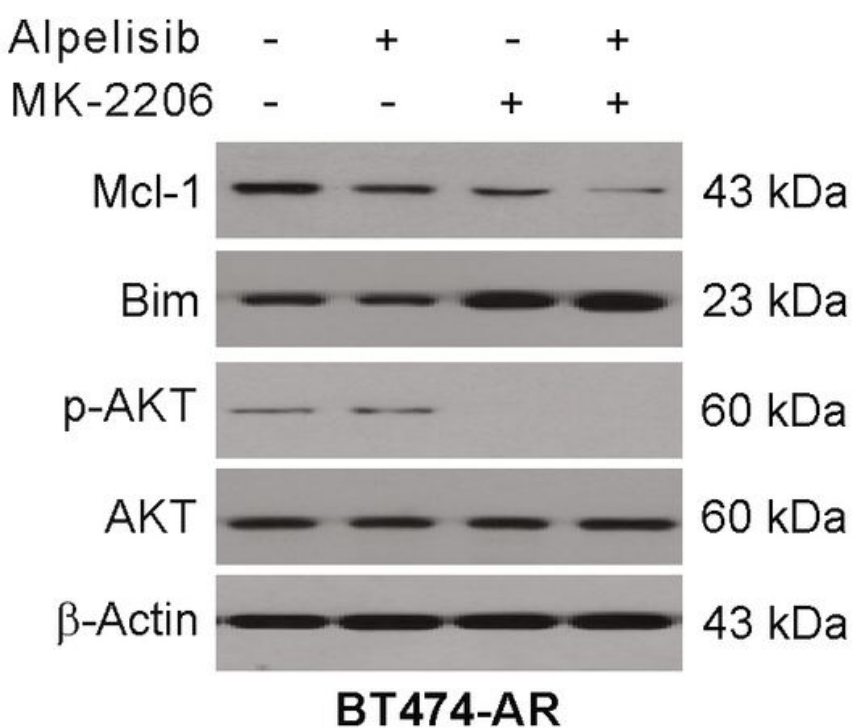

C

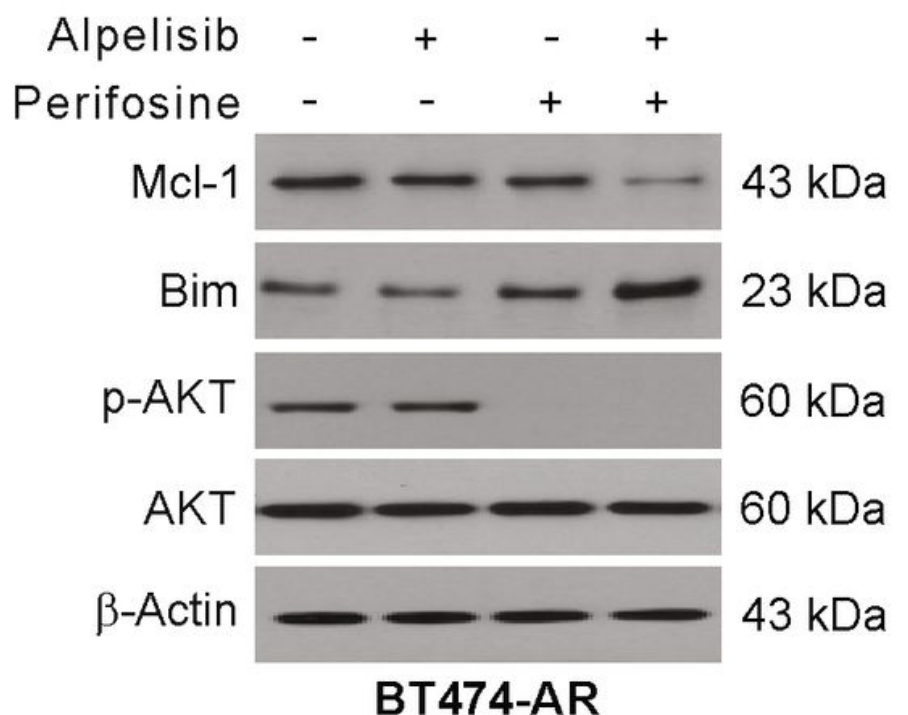

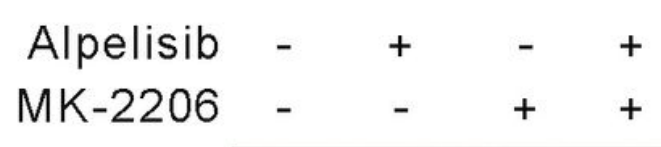

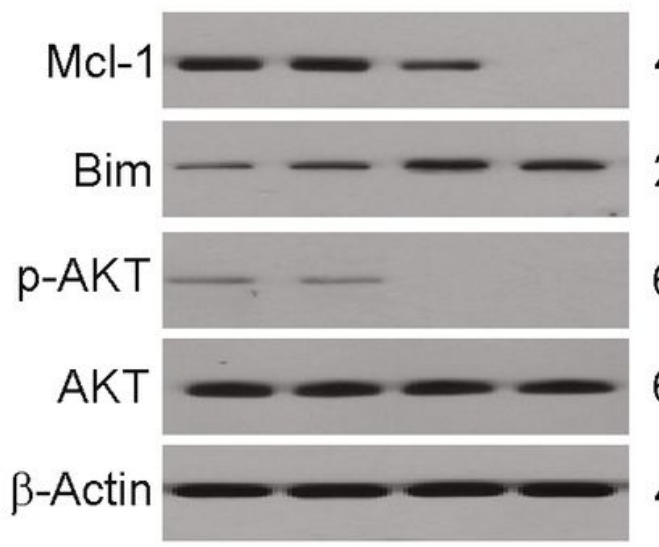

$43 \mathrm{kDa}$

$23 \mathrm{kDa}$

$60 \mathrm{kDa}$

$60 \mathrm{kDa}$

$43 \mathrm{kDa}$

361-AR

D

\section{Figure 4}

AKT inhibitors decrease Mcl-1, p-AKT and increased Bim level. (A) BT474-AR cells were treated with $1 \mu \mathrm{M}$ alpelisib, $1 \mu \mathrm{M}$ MK-2206, or their combination. The indicated protein level was analyzed by Western blotting. (B) MDA-MB-361-AR cells were treated with1 $\mu \mathrm{M}$ alpelisib, $1 \mu \mathrm{M}$ MK-2206, or their combination. The indicated protein level was analyzed by Western blotting. (C) BT474-AR cells were treated with $1 \mu \mathrm{M}$ alpelisib, $1 \mu \mathrm{M}$ Perifosine, or their combination. The indicated protein level was analyzed by Western blotting. (D) MDA-MB-361-AR cells were treated with1 $\mu \mathrm{M}$ alpelisib, $1 \mu \mathrm{M}$ Perifosine, or their combination. The indicated protein level was analyzed by Western blotting. 
A

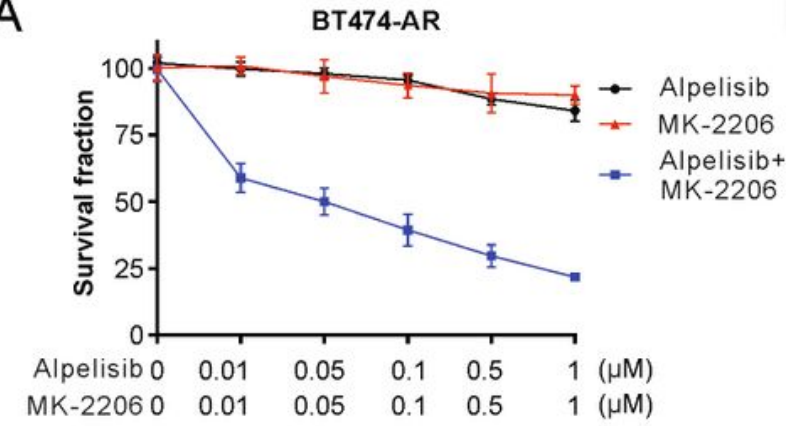

C

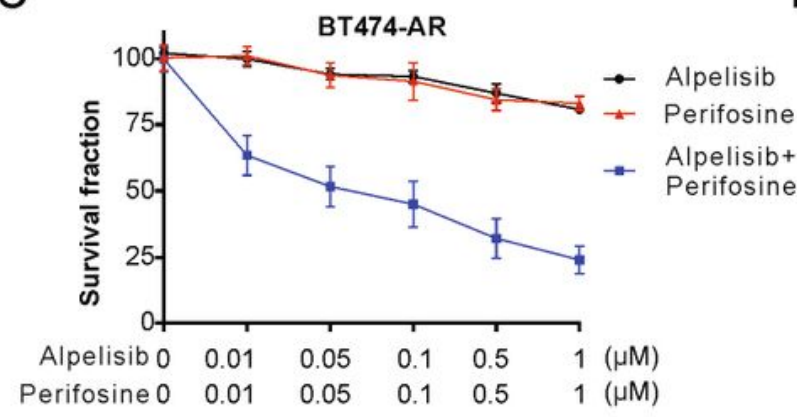

E

BT474-AR

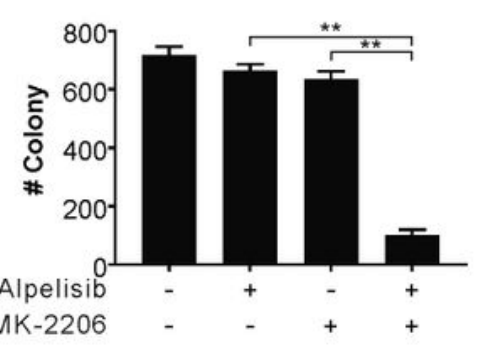

G
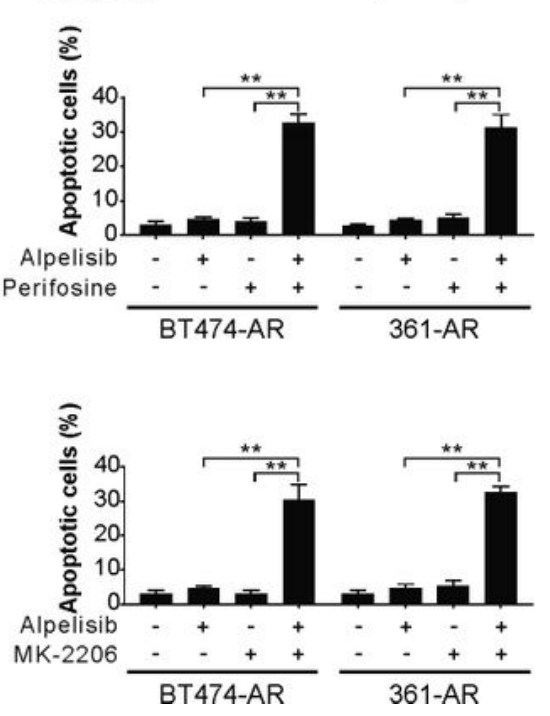

B

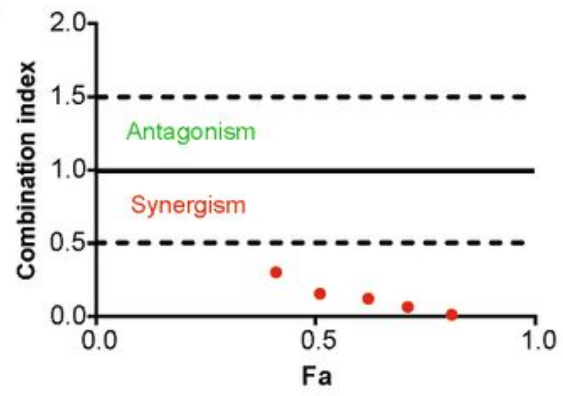

D

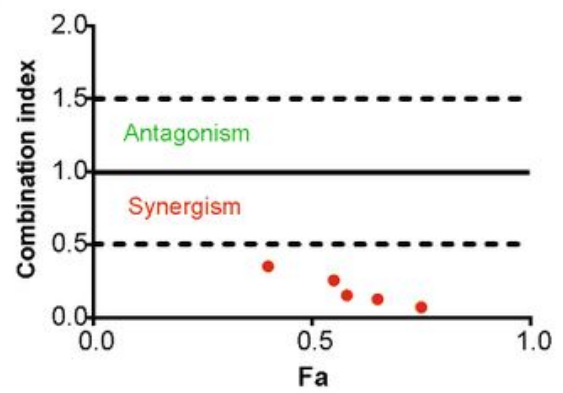

F

BT474-AR

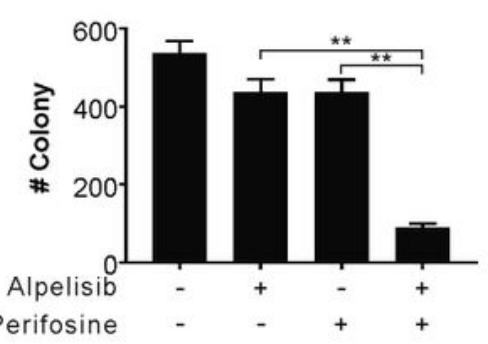

$\mathrm{H}$

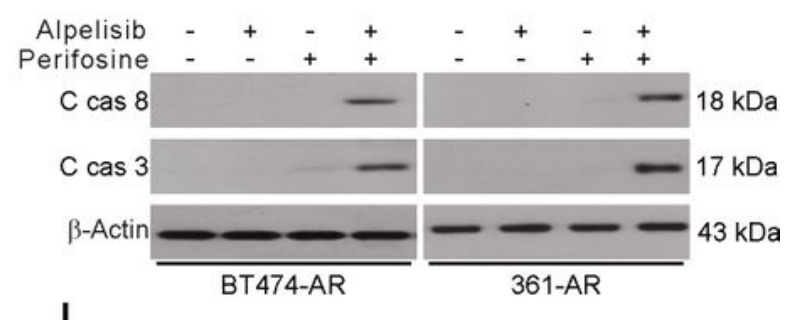

J

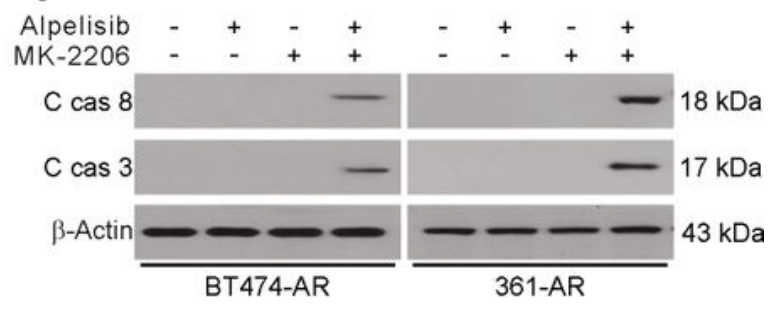

\section{Figure 5}

The combination of AKT inhibitors with alpelisib effectively inhibits the growth and induces apoptosis of alpelisib-resistant breast cancer cell lines. (A)-(B) Sensitivity of BT474-AR cells to alpelisib, MK-2206, or their combination. Survival fraction (left) and the $\mathrm{Cl}$ (right) are shown. Fa, fraction affected. (C)-(D) Sensitivity of BT474-AR cells to alpelisib, Perifosine, or their combination. Survival fraction (left) and the $\mathrm{Cl}$ (right) are shown. Fa, fraction affected. (E) BT474-AR cells were treated with $1 \mu \mathrm{M}$ alpelisib, $1 \mu \mathrm{M}$ MK- 
2206 , or their combination. After 14 days, the plates were stained for cell colonies with crystal violet dye. The colonies were then counted. (F) BT474-AR cells were treated with $1 \mu \mathrm{M}$ alpelisib, $1 \mu \mathrm{M}$ Perifosine, or their combination. After 14 days, the plates were stained for cell colonies with crystal violet dye. The colonies were then counted. (G) BT474-AR or MDA-MB-361-AR cells were treated with $1 \mu \mathrm{M}$ alpelisib, $1 \mu \mathrm{M}$ Perifosine, or their combination for 48 hours. Apoptosis was analyzed by flow cytometry. (H) BT474-AR or MDA-MB-361-AR cells were treated with $1 \mu \mathrm{M}$ alpelisib, $1 \mu \mathrm{M}$ Perifosine, or their combination for 48 hours. The indicated protein level was analyzed by Western blotting. (I) BT474-AR or MDA-MB-361-AR cells were treated with $1 \mu \mathrm{M}$ alpelisib, $1 \mu \mathrm{M}$ MK-2206, or their combination for 48 hours. Apoptosis was analyzed by flow cytometry. (J) BT474-AR or MDA-MB-361-AR cells were treated with $1 \mu \mathrm{M}$ alpelisib, $1 \mu \mathrm{M}$ MK-2206, or their combination for 48 hours. The indicated protein level was analyzed by Western blotting. The results were expressed as the means \pm SD of three independent experiments. ${ }^{* *}, P<0.01$.

A

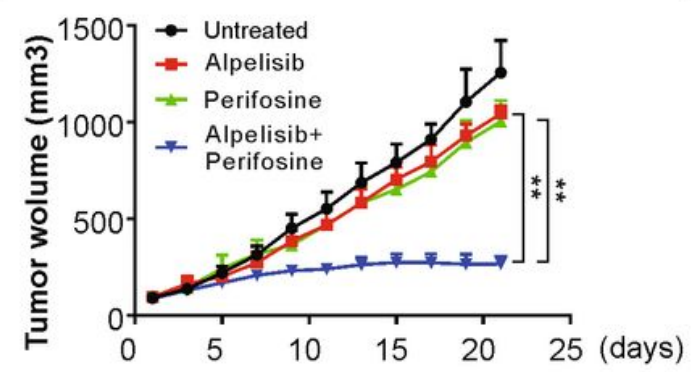

D

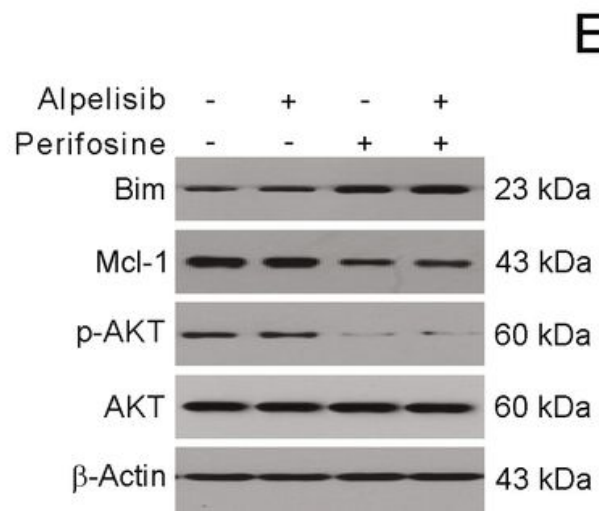

$E$
B

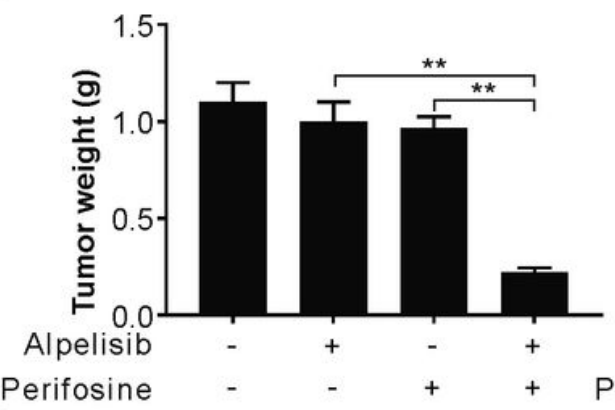

C

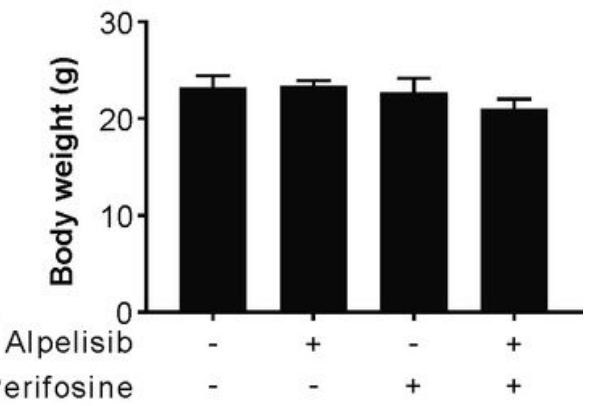

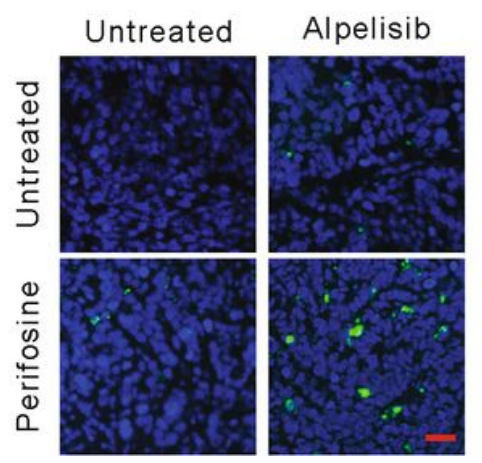

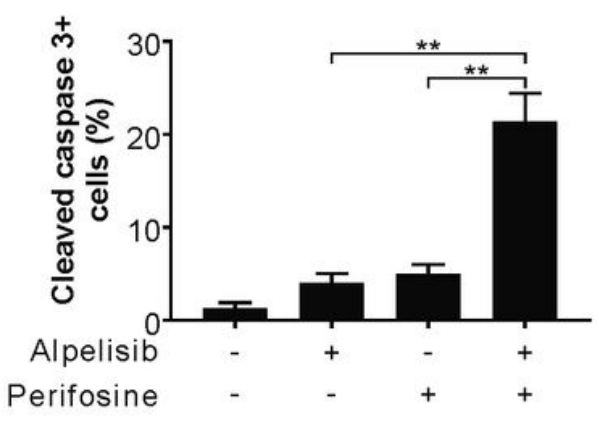

\section{Figure 6}

Perifosine sensitizes alpelisib-induced tumor growth in vivo. (A) BT474-AR xenografts were treated with alpelisib, Perifosine, or their combination. Tumor volume at indicated time points after treatment was calculated and plotted with $p$ values, $n=6$ in each group. (B) At the end of the treatment, the mice were sacrificed and the tumors were removed and weighed. (C) Mouse body weights were also compared. (D) Whole protein cell lysates were prepared randomly from 3 tumors in each group for Western blotting to detect the indicated proteins. (E) Tissue sections were analyzed by active caspase 3 staining. Left, representative staining pictures, scale bar: $25 \mu \mathrm{m}$; Right, active caspase 3-positive cells were counted and plotted.

\section{Supplementary Files}


This is a list of supplementary files associated with this preprint. Click to download.

- Supplementaryfile.docx

- Supplementaryfile.docx

- Supplementaryfile.docx 